\title{
Pyrylium Salts: Selective Reagents for the Activation of Primary Amino Groups in Organic Synthesis
}

Yue Pang

Daniel Moser

Josep Cornella

Synthesis 2020, 52, 489.

In the originally uploaded e-first version of this short review, an important reference, 55e, was missing. This reference was added, together with a short paragraph describing the chemistry, to the eighth page of an updated version, published on $15^{\text {th }}$ of October. 